\title{
Global contrasts between oceanic cycling of cadmium and phosphate
}

\author{
SAEED ROSHAN ${ }^{12}$, TIM DEVRIES $^{12}$
}

${ }^{1}$ Deprtment of Geography, University of California, Santa Barbara, CA, USA 93106

${ }^{2}$ Earth Research Institute, University of California, Santa Barbara, CA, USA 93106

Cadmium (Cd) is a trace metal whose distribution in the ocean bears a remarkable resemblance to that of phosphate (PO4). This resemblance suggests that the Cd incorporated in microfossils may hold information about the past ocean circulation and biology, leading to the development of $\mathrm{Cd}$ as a tracer for paleoceanographic applications. Nonetheless, the origin of both the linear correlation and the subtle deviations between concentrations of $\mathrm{Cd}$ and PO4 are not known, complicating the utilization of $\mathrm{Cd}$ as a paleo-tracer. In this study, we use the available Cd observations and an Artificial Neural Network to produce a seasonal climatology of dissolved $\mathrm{Cd}$, that also reproduces the observed subtle deviations between $\mathrm{Cd}$ and $\mathrm{PO} 4$ distributions. We use this $\mathrm{Cd}$ climatology and an available PO4 climatology, along with an ocean circulation inverse model, to diagnose the biogeochemical sources and sinks of dissolved Cd and PO4. We find large difference between the biogeochemical cycling of $\mathrm{Cd}$ and $\mathrm{P}$ in both the surface and subsurface ocean, and show that the quasi-linear $\mathrm{Cd}-\mathrm{PO} 4$ relationship is results from the competing effects of variability in the $\mathrm{Cd}: \mathrm{P}$ export ratio and $\mathrm{Cd}: \mathrm{P}$ remineralization ratio. The Cd:P export ratio varies as a Monod function of $\mathrm{Cd}$ concentration, reaching highest values in the Southern Ocean. This effect alone imparts substantially non-linearity to the Cd:PO4 relationship, but is compensated for by deeper remineralization and excess removal of $\mathrm{Cd}$ in the deep ocean relative to $P$. Nevertheless, the combination of these processes is not precisely balanced, inducing a "kink" or "concavity" in the Cd: PO4 relationship. Our results reveal key distinctions between the cycling of $\mathrm{Cd}$ and $\mathrm{P}$ that should be taken into account when interpreting paleoceanographic records. 